\title{
A Prospective Crossover Trial Comparing Performance and in vitro Platelet Quality of Three New Apheresis Devices with Current Equipment
}

\author{
Susanne M. Picker Stela M. Radojska Birgit S. Gathof \\ Transfusionsmedizin, Klinikum der Universität zu Köln, Germany
}

\section{Key Words}

PLT apheresis collection - Triple-dose PLT collection . High-dose PLT collection - Baxter Fenwal Amicus . Gambro Trima Accel · Haemonetics MCS+

\section{Summary}

Background: To improve productivity of automated platelet (PLT) collection, the industry has introduced new instruments or modifications to existing equipment. Materials and Methods: Data obtained from 8 regular PLT apheresis donors randomized to double- (DDC) or triple-dose PLT collection (TDC) with the BAXTER Amicus (AM), the HAEMONETICS MCS Plus (MCS+), and the GAMBRO Trima Accel (TA) were evaluated focusing on duration, citrate infusion and product quality, and statistically compared to data obtained from the same donors during DDC on our current equipment (GAMBRO Trima V4 (TV4) and Spectra LRS-Turbo V7 (SPC)). Results: All units were sufficiently leukoreduced to below $1 \times 10^{6}$ white blood cells (WBCs). Apart from statistically significant lower $\mathrm{pH}$ values and higher CD62P expression observed with AM units, no differences in in vitro function were noted during storage. Compared to our current equipment, the new devices had advantages for whole blood processed, and, except for MCS+, for needle time, collection volume, collection rate, and collection efficiency. PLT yield and processing time were equivalent, except for TA which was the fastest machine. MCS+ was the slowest device owing to statistically significant lower draw and collection rates which were, however, compensated by fewer citrate reactions due to only moderate citrate infusion rates. Conclusion: Despite equal or better efficiencies, collection procedures with the new devices did not automatically increase the number of units per day, particularly if quick donation was counteracted by long overall performance (AM). TA was the fastest and hence offered the highest potential to optimize productivity. MCS+ showed better donor comfort, as reflected by lower draw and citrate infusion rates, but was also the slowest.

\author{
Schlüsselwörter \\ Thrombozytapherese - Hochdosis-Plättchen-Sammlung · \\ Triple-Dosis-Plättchen-Sammlung · Baxter Fenwal Amicus \\ Gambro Trima Accel · Haemonetics MCS+
}

\section{Zusammenfassung}

Hintergrund: Um die Produktivität automatischer Thrombozytensammlung zu steigern, hat die Industrie neue Instrumente eingeführt bzw. bestehendes Equipment modifiziert. Material und Methoden: Daten, die mit den Zellseparatoren BAXTER Amicus (AM), HAEMONETICS MCS Plus (MCS+), und GAMBRO Trima Accel (TA) von 8 Spendern nach vorheriger Randomisierung zur Doppel- oder Triple-Dosis-Sammlung hinsichtlich Prozessdauer, Sammeleffizienz, Zitratbelastung und In-vitro-Funktion erhoben wurden, wurden mit Daten derselben Spender an unseren herkömmlichen Geräten (GAMBRO Trima V4 (TV4), Spectra LRS-Turbo V7 (SPC)) verglichen. Ergebnisse: Alle Prüfparameter der untersuchten Einheiten waren richtlinienkonform und unterschieden sich abgesehen von statistisch signifikant niedrigeren $\mathrm{pH}$ Werten und höherer CD62P-Expression bei AM-Einheiten nicht in ihrer In-vitro-Funktion. Im Vergleich zu unseren herkömmlichen Geräten, vor allem der SPC, besaßen die neuen Geräte Vorteile beim prozessierten Blutvolumen, und, außer MCS+, bei der Nadelzeit, dem Sammelvolumen, der Sammelrate und -effizienz. Dagegen waren der Ertrag und die Gesamtprozesszeit gleichwertig, mit Ausnahme des TA, welches das schnellste Gerät war. Aufgrund statistisch signifikant niedriger Entnahme- und Sammelraten, war MCS+ das langsamste Gerät, zeigte jedoch weniger Zitratnebenwirkungen infolge niedriger Zitratinfusionsraten. Schlussfolgerung: Trotz besserer Sammeleffizienz steigerten die neuen Geräte nicht automatisch die Produktivität, vor allem, wenn eine lange Gesamtprozesszeit den Vorteil einer schnellen Spende wieder aufhob (AM). Aufgrund seiner Schnelligkeit bot TA das größte Potential zur Produktivitätssteigerung. MCS+Verfahren waren aufgrund niedrigerer Entnahme und Zitratinfusionsraten zwar komfortabler, gleichzeitig jedoch auch die langsamsten.

\section{KARGER \\ (c) 2006 S. Karger GmbH, Freiburg \\ Fax +497614520714




\section{Introduction}

Medical development including stem cell transplantation results in the steady increase of transfusion demands, particularly platelets (PLTs) [1]. Concurrently, the recruitment and retention of voluntary and eligible blood donors is becoming increasingly difficult due to aging of the population and more stringent exclusion criteria for blood donation. High-dose PLT apheresis enabling the collection of multiple units in a single donation may have the potential to counteract this problem and to reduce both, costs and risks inherent with allogenic transfusion [2-5]. As a result, the industry has introduced new apheresis systems or modified existing equipment for better productivity and donor safety. Even in the absence of severe side effects during automated collection procedures [6], the latter has been of growing interest as relatively high rates of citrate reactions have been described recently with new devices [7]. Apart from efficiency and donor comfort, apheresis systems should guarantee consistent product quality, particularly in terms of reliable attainment of the targeted PLT dose and consistent leukoreduction, performed either by filtration or by physical separation, utilizing different cell densities of PLTs and mononuclear cells $[8,9]$. The white blood cell (WBC) purity obtained by the latter techniques is reported to be more consistent than that obtained upon filtration $[10,11]$. However, according to a single report [12], up to $5 \%$ of apheresis PLTs are reported to fail the cut-off standard of residual WBCs for unknown reasons. Provided that new apheresis systems can combine high efficiency with quick performance, few adverse events, moderate blood cell loss and high product quality, blood centers could aim more intensively at high-dose PLT apheresis. Therefore, the aim of this study was to prospectively compare the performance of 3 newly introduced apheresis devices with currently used systems during high-dose PLT apheresis based on the same healthy blood donors and similar target endpoints.

\section{Materials and Methods}

\section{Donors}

8 healthy regular PLT apheresis donors (1 female, 7 males; $38.2 \pm 8.2$ years of age) with at least $200 \times 10^{3}$ PLTs per $\mu$, were selected from our donor pool. All participants gave informed written consent and met the German and European guidelines and requirements for cytapheresis [13-15]. As (inter)national recommendations for high-dose, especially triple-dose PLT donors are lacking, donors with known high PLT counts due to a large body surface (height $\geq 1.75 \mathrm{~m}$, weight $\geq 75 \mathrm{~kg}$, total blood volume $\geq 5.0 \mathrm{l}$ ) were selected from our regular PLT donor pool. 4 donors were randomly assigned to double-dose PLT collection (DDC), the remaining 4 to tripledose PLT collection (TDC) with each of the following 3 systems: the Amicus (AM, BAXTER Transfusion Therapies, Deerfield, IL, USA), the MCS Plus (MCS+, HAEMONETICS Corp., Braintree, MA, USA), and the Trima Accel (TA, GAMBRO BCT, Lakewood, CO, USA) following the manufacturers' instructions. There were at least 2 weeks between every donation by the same donor. Results obtained with the study devices were compared to data obtained during DDC on our current equip- ment (TV4, GAMBRO Trima V4, and SPC, GAMBRO Spectra LRSTurbo V7) in routine use (before the study, we did not perform TDC). For DDC and TDC donors, the mean body weight was $84.9 \pm 9.4$ and $86.5 \pm$ $13.8 \mathrm{~kg}$, the mean height was $1.84 \pm 0.08$ and $1.83 \pm 0.13 \mathrm{~m}$, the mean body surface was $2.08 \pm 0.18$ and $2.10 \pm 0.22 \mathrm{~m}^{2}$, the mean body mass index was $25.0 \pm 1.5$ and $25.9 \pm 2.9 \mathrm{~kg} / \mathrm{m}^{2}$, and the mean total blood volume was $5631.8 \pm 682.4$ and $5680.2 \pm 796.0 \mathrm{ml}$, respectively. The pre-procedure hematologic variables were similar among the study groups: hematocrit (Hct): $44.2 \pm 2.4 \%$ (DDC) and $43.5 \pm 2.0 \%$ (TDC); PLTs: $278.5 \pm 22.5$ (DDC) and $319.8 \pm 34.5 \times 10^{9} / 1($ TDC) WBCs: $7.4 \pm 2.1$ (DDC) and $7.3 \pm$ $1.5 \times 10^{9} / 1$ (TDC), respectively. Side effects and any necessary treatments were documented by the operator.

\section{Platelet Apheresis Systems}

Main characteristics and technical features of the PLT apheresis systems used in this study are described in more detail elsewhere in the literature [16-21]. MCS + was the only device operating a discontinuous separation technique, while all other instruments were continuous operating systems. The MCS + machine was set to perform a minimum of 7-8 cycles for DDC and TDC, respectively. Unlike TA, operating at a fixed anticoagulant ratio from process start, TV4 and SPC used progressively increasing ratios of whole blood to anticoagulant (table 1) within the first 15 (TV4) to 20 min (SPC). In contrast to SPC, no priming was needed for Trima procedures. Except for AM and SPC (dual-needle sets), all donations were performed with single-needle sets and intermittent flow. Draw and return rates were determined by the instrument according to donor size unless access problems or donor comfort required adjustments. Acid-citrate-dextrose-A (ACD-A) was used as anticoagulant for all collections. Settings, definitions, and formulas used are presented in tables 2 and 3.

\section{Target Endpoints}

Target PLT yields of 5.5-6.0 $\times 10^{11}$ PLTs $\left(\right.$ DDC) and 7.5-8.0 $\times 10^{11}$ PLTs (TDC) in up to $100 \mathrm{~min}$ (DDC) and $120 \mathrm{~min}$ (TDC) were used for DDC and TDC, respectively. To target the endpoints, the yield predictors in each system were used according to manufacturers' recommendations. Once pre-procedure PLT counts were obtained, they were entered into the predictor. If the range of target yield could be obtained in different donation times or cycle numbers, that target was used, getting as close as possible to the lower limit of PLT yield in the minimum donation time or numbers of cycles.

\section{In vitro Testing}

Peripheral blood samples were obtained before and after each apheresis procedure and monitored for blood cell counts and hematocrit. The preprocedure samples were withdrawn immediately before procedure by direct venipuncture. Post-procedure samples were drawn from the access site after the end of reinfusion with a $5 \mathrm{ml}$ waste sample removed.

After splitting and sterile connection of an additional storage bag, each product was weighted to confirm that it had been divided evenly. All preand post-procedure hematological parameters as well as product measurements were performed on the same automated analyzer (Sysmex K1000, Sysmex, Hamburg, Germany). Prior to laboratory analysis, PLTs were stored for 10-12 h under agitation in a PLT incubator (Helmer Labs, distributed by Baxter Germany, Unterschleissheim, Germany) for adequate resuspension after collection. Agitation started immediately after collection without a resting period. Contaminating WBCs and red blood cells (RBCs) were evaluated by flow cytometry (FACScan Becton Dickinson, Heidelberg, Germany) immediately after collection using the LeukoCOUNT $^{\circledR}$ test kit and anti-glycophorin A in TruCOUNT ${ }^{\circledR}$ tubes (Becton Dickinson), respectively. In vitro quality of PLTs was determined in 2-day intervals until the end of storage (day 5) as described previously [22]. Samples for blood gas analysis, lactate and glucose measurements were analyzed immediately in an automated analyzer (ABL System 625, Radiometer, Willich, Germany). $\mathrm{pH}$ values were measured at $37^{\circ} \mathrm{C}$ and corrected to $22{ }^{\circ} \mathrm{C}$ using the formula given by the manufacturer: $\mathrm{pH}_{22}{ }^{\circ} \mathrm{C}=$ 
Table 1. Instrument settings

\begin{tabular}{|c|c|c|c|c|c|}
\hline Settings & Amicus & $\mathrm{MCS}+$ & Trima Accel & Spectra LRS-Turbo & Trima \\
\hline Software version & 2.51 & LDPC.4 & 5.0 & 7.0 & 4.0 \\
\hline Set code REF & R4R2340 & 994CFE & 80300 & 70310 & 80000 \\
\hline Citrate infusion rate, $\mathrm{mg} / \mathrm{kg} / \mathrm{min}$ & 1.25 & 1.5 & $\begin{array}{l}2.0-2.4(=1.0- \\
\left.1.2 \mathrm{ml} / \mathrm{min} / 1 \mathrm{TBV}^{\mathrm{a}}\right)\end{array}$ & $\begin{array}{l}2.0(=1.0 \mathrm{ml} / \mathrm{min} / 1 \\
\text { TBV })\end{array}$ & $\begin{array}{l}2.0-2.4(=1.0- \\
\left.1.2 \mathrm{ml} / \mathrm{min} / 1 \mathrm{TBV}^{\mathrm{a}}\right)\end{array}$ \\
\hline Anticoagulant ratio & $\begin{array}{l}\text { 10:1 } \\
\text { (no ramping) }\end{array}$ & $\begin{array}{l}\text { 9:1 } \\
\text { (no ramping) }\end{array}$ & $\begin{array}{l}\text { 11:1 } \\
\text { (no ramping) }\end{array}$ & $\begin{array}{l}13: 1 \\
\text { (ramping from 8:1) }\end{array}$ & $\begin{array}{l}\text { 11:1 } \\
\text { (ramping from } 7: 1 \text { ) }\end{array}$ \\
\hline Maximum inlet (draw) rate, $\mathrm{ml} / \mathrm{min}$ & 70 & 90 & $70-120^{\mathrm{b}}$ & 140 & $70-120^{\mathrm{b}}$ \\
\hline Maximum return rate, $\mathrm{ml} / \mathrm{min}$ & 70 & 120 & $130-250^{\mathrm{c}}$ & 250 & $130-250^{\mathrm{c}}$ \\
\hline Minimum draw pressure limit, $\mathrm{mmHg}$ & -250 & $-50^{\mathrm{d}}$ & -250 & -250 & -250 \\
\hline Maximum return pressure limit, $\mathrm{mmHg}$ & 450 & $90^{\mathrm{d}}$ & 310 & 310 & 310 \\
\hline Maximum cycle volume, $\mathrm{ml}$ & 250 & $450-500^{\mathrm{e}}$ & 196 & 131 & 230 \\
\hline
\end{tabular}

$\mathrm{LRF}=$ Leukoreduction filter; $\mathrm{TBV}=$ total blood volume.

${ }^{\mathrm{a} C}$ Corresponding to $\mathrm{AC}$ management 4 , decreases with increasing processing time and decreasing flow rate.

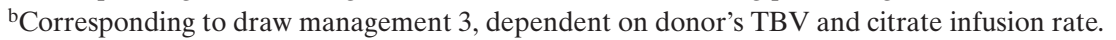

${ }^{\mathrm{c} C o r r e s p o n d i n g ~ t o ~ r e t u r n ~ m a n a g e m e n t ~} 2$.

${ }^{\mathrm{d}}$ According to personnel communication with the manufacturer.

${ }^{e}$ Dependent on donor hematocrit.

Table 2. Definitions, abbreviations, and formulas used

\begin{tabular}{|c|c|c|}
\hline Parameter & Abbreviation & Definition \\
\hline Needle time, $\min$ & NT & $\mathrm{DT}(\mathrm{min})^{\mathrm{a}}+$ reinfusion time $(\mathrm{min})+$ post-procedure control sample $(\mathrm{min})$ \\
\hline Draw rate, $\mathrm{ml} / \mathrm{min}$ & $\mathrm{DR}$ & mean blood flow $=\left[\mathrm{WBP}(\mathrm{ml})+\right.$ citrate volume $\left.(\mathrm{ml})^{\mathrm{b}}\right] \div \mathrm{DT}(\mathrm{min})^{\mathrm{a}}$ \\
\hline ACD-A infused, $\mathrm{ml}$ & ACD-A ${ }^{\text {inf }}$ & ACD-A used ${ }^{a}-$ ACD-A of products ${ }^{c}$ \\
\hline Citrate infusion rate, $\mathrm{ml} / \mathrm{min} / \mathrm{l}$ of donor's blood volume & CIR & ACD-A $\mathrm{A}^{\mathrm{inf}} / \mathrm{DT}(\mathrm{min})^{\mathrm{a}} \div$ total blood volume of donor $(\mathrm{ml}) / 1000$ \\
\hline Whole blood processed, $\mathrm{ml}$ & WBP & volume processed from start until reinfusion $(\mathrm{ml})^{\mathrm{a}}$ - citrate volume $(\mathrm{ml})^{\mathrm{b}}$ \\
\hline Time for a $2.5 \times 10^{11}$ standard dose, $\min$ & TSD 2.5 & $2.5 \times 10^{11} \times \mathrm{PT}(\mathrm{min}) / \mathrm{PLT}$ yield $\left(\times 10^{11}\right)$ \\
\hline Collection rate, $\times 10^{11} / \mathrm{min}$ & CR & PLT yield $\left(\times 10^{11}\right) / \mathrm{DT}(\mathrm{min})^{\mathrm{a}}$ \\
\hline Mean PLT count, $\times 10^{6} / \mathrm{ml}$ & MPC & [pre-procedure PLT count $\left(\times 10^{6}\right)+$ post-procedure PLT count $\left.\left(\times 10^{6}\right)\right] / 2$ \\
\hline Collection efficiency, \% & $\mathrm{CE}$ & PLT yield $\left(\times 10^{11}\right) \times 10^{5}\left[\mathrm{MPC}\left(\times 10^{6} / \mathrm{ml}\right) \times(\mathrm{WBP}(\mathrm{ml})-\mathrm{X}(\mathrm{ml}))\right] \times 100$ \\
\hline
\end{tabular}

$\mathrm{PT}=$ Processing time $; \mathrm{ACD}-\mathrm{A}=; \mathrm{PLT}=$ platelet $\mathrm{RBC}=$ red blood cell.

$\mathrm{X}=$ blood volume processed without harvest of PLTs; $X=30$ (Amicus, Spectra), $X=20$ (MCS+ to purge priming solution), $X=200$ (Trima V4) and X $=400(\mathrm{TA}$, to ensure the RBC plasma interface [23]).

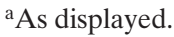

${ }^{\mathrm{b}}$ As defined by the anticoagulant ratio.

cAs measured photometrically (Ultrospec 1000, Pharmacia Biotech, Cambridge, UK) using kit technology (Citric acid, UV method, R-Biopharm AG, Darmstadt, Germany).

$\mathrm{pH}_{37}{ }^{\circ} \mathrm{C}-\left[0,0146+0.0065 \times\left(\mathrm{pH}_{37}^{\circ} \mathrm{C}-7.40\right)\right] \times(22-37)$. PLT activation was determined by flow cytometry (FACScan, Becton Dickinson) using the CD62P monoclonal antibody for detection of p-selectin on the membrane surface. Labeling of PLTs was performed with anti-CD61PerCP and anti-CD41Cy.PerCP. The proportion of activated PLTs expressing pselectin was defined by a threshold set at the upper limit of fluorescence for the negative control sample. Hypotonic shock response (HSR) was performed as described by Fantl [23] using a spectrophotometer (Photometer 1101, Eppendorf, Hamburg, Germany). The HSR was assessed as the percentage of a $100 \%$ recovery designated by a dilution of the same PLTs in $0.9 \% \mathrm{NaCl}$. Aggregation maximum was measured using the technique described and developed by Born [24]. Aggregometry was performed on an aggregometer (APACT, LAbor, Ahrensburg, Germany, APACT software version 1.4, October 1999) using ristocetin $1.5 \mu \mathrm{mol} / \mathrm{l}$
(Diamed, Ottobrunn, Germany). Before exposure to hypotonic shock and stimulation of aggregation, PLT concentration was adapted to $250,000 / \mu \mathrm{l}$ by addition of $\mathrm{AB}$ plasma.

\section{Statistical Analysis}

Results are presented as mean \pm standard deviation. Statistical comparisons were made with the Kruskal-Wallis test using commercially available software (SPSS 11.0 for Windows, SPSS Software GmbH, Munich, Germany). In the case of significant differences, post-hoc paired comparisons were made with the Mann-Whitney U test after the significance level had been adjusted according to Bonferoni. $p$ values of below 0.017 and 0.005 were considered significant for 3 and 10 comparisons, respectively. 
Table 3. Donor safety and comfort

\begin{tabular}{|c|c|c|c|c|c|c|}
\hline & Amicus & $\mathrm{MCS}+$ & Trima Accel & $\begin{array}{l}\text { Spectra LRS- } \\
\text { Turbo V7 }\end{array}$ & Trima V4 & $\mathrm{p}$ value \\
\hline \multicolumn{7}{|c|}{ Collection procedures, $\mathrm{n}$} \\
\hline DDC & 4 & 4 & 4 & 4 & 4 & \multirow[t]{2}{*}{4} \\
\hline TDC & 4 & 4 & 4 & 0 & 0 & \\
\hline \multicolumn{7}{|c|}{ PLT loss, \% initial value } \\
\hline DDC & $36.2 \pm 3.8$ & $32.9 \pm 5.5$ & $29.9 \pm 6.6$ & $31.4 \pm 10.7$ & $33.5 \pm 6.9$ & 0.259 \\
\hline TDC & $41.5 \pm 7.1$ & $35.8 \pm 5.5$ & $31.7 \pm 7.3$ & - & - & 0.068 \\
\hline Total procedures & $38.8 \pm 6.0$ & $34.4 \pm 5.3$ & $30.8 \pm 6.5$ & $31.4 \pm 10.7$ & $33.5 \pm 6.9$ & 0.211 \\
\hline \multicolumn{7}{|l|}{ Needle time, $\min$} \\
\hline DDC & $64.7 \pm 10.5^{\mathrm{c}, \mathrm{d}}$ & $107.7 \pm 4.1^{a, b, d, e}$ & $62.6 \pm 7.7^{\mathrm{c}, \mathrm{d}}$ & $82.8 \pm 7.6^{\mathrm{a}, \mathrm{b}, \mathrm{c}}$ & $74.6 \pm 10.2^{\mathrm{c}}$ & $<0.001$ \\
\hline TDC & $85.3 \pm 5.7^{\mathrm{c}}$ & $118.2 \pm 13.8^{a, b}$ & $77.2 \pm 16.3^{c}$ & - & - & $<0.001$ \\
\hline Total procedures & $75.0 \pm 13.5^{\mathrm{c}}$ & $112.9 \pm 13.5^{a, b, d, e}$ & $69.9 \pm 14.1^{\mathrm{c}}$ & $82.8 \pm 7.6^{c}$ & $74.6 \pm 10.2^{\mathrm{c}}$ & $<0.001$ \\
\hline \multicolumn{7}{|l|}{ Draw rate, $\mathrm{ml} / \mathrm{min}$} \\
\hline DDC & $69.4 \pm 2.0^{\mathrm{c}}$ & $41.3 \pm 4.2^{a, b, d, e}$ & $67.0 \pm 5.6^{\mathrm{c}}$ & $68.6 \pm 8.4^{c}$ & $62.9 \pm 8.5^{\mathrm{c}}$ & $<0.001$ \\
\hline $\mathrm{TDC}$ & $65.5 \pm 3.2^{\mathrm{c}}$ & $43.3 \pm 2.0^{a, b}$ & $68.3 \pm 9.3^{c}$ & - & - & $<0.001$ \\
\hline Total procedures & $67.4 \pm 3.2^{c}$ & $43.3 \pm 3.2^{a, b, d, e}$ & $67.7 \pm 7.2^{\mathrm{c}}$ & $68.6 \pm 8.4^{\mathrm{c}}$ & $62.9 \pm 8.5^{\mathrm{c}}$ & $<0.001$ \\
\hline \multicolumn{7}{|c|}{ Whole blood processed, ml } \\
\hline DDC & $3,707 \pm 569^{d}$ & $3,711 \pm 308^{\mathrm{d}}$ & $3,449 \pm 300^{\mathrm{d}}$ & $4,751 \pm 437^{a, b, c, e}$ & $3,698 \pm 364^{\mathrm{d}}$ & $<0.001$ \\
\hline TDC & $4,731 \pm 458$ & $4,296 \pm 427$ & $4,406 \pm 411$ & - & - & 0.068 \\
\hline Total procedures & $4,219 \pm 727$ & $4,003 \pm 465$ & $3,928 \pm 611$ & $4,752 \pm 437^{\mathrm{e}}$ & $3,698 \pm 364^{\mathrm{d}}$ & 0.030 \\
\hline \multicolumn{7}{|c|}{ Citrate infusion rate, $\mathrm{ml} / \mathrm{min} / \mathrm{l}$} \\
\hline DDC & $1.01 \pm 0.07^{\mathrm{c}}$ & $0.77 \pm 0.16^{\mathrm{a}, \mathrm{b}, \mathrm{d}}$ & $1.09 \pm 0.07^{\mathrm{c}, \mathrm{e}}$ & $1.06 \pm 0.04^{\mathrm{c}, \mathrm{e}}$ & $0.93 \pm 0.05^{\mathrm{b}, \mathrm{d}}$ & $<0.001$ \\
\hline TDC & $0.90 \pm 0.16$ & $0.80 \pm 0.04^{\mathrm{b}}$ & $0.95 \pm 0.07^{\mathrm{c}}$ & - & - & 0.026 \\
\hline Total procedures & $0.95 \pm 0.13$ & $0.78 \pm 0.11^{\mathrm{b}, \mathrm{d}}$ & $1.02 \pm 0.10^{\mathrm{c}}$ & $1.06 \pm 0.04^{\mathrm{c}, \mathrm{e}}$ & $0.93 \pm 0.05^{\mathrm{d}}$ & $<0.001$ \\
\hline \multicolumn{7}{|l|}{$\mathrm{Ca}^{++}$needed, n (\%) } \\
\hline DDC & $3(75.0)$ & $0(0.0)$ & $3(75.0)$ & $6(75.0)$ & $3(37.5)$ & - \\
\hline TDC & $2(50.0)$ & $1(25.0)$ & $3(75.0)$ & - & - & - \\
\hline Total procedures & $5(62.5)$ & $1(12.5)$ & $6(75.0)$ & $6(75.0)$ & $3(37.5)$ & - \\
\hline
\end{tabular}

DDC $=$ Double-dose PLT collection $;$ TDC $=$ triple-dose PLT collection; $\mathrm{PLT}=$ platelet

Significant to: ${ }^{\mathrm{a}}$ Amicus, ${ }^{\mathrm{b}}$ Trima Accel, ${ }^{\mathrm{c} M C S}+,{ }^{\mathrm{d}}$ Spectra LRS-Turbo V7, ${ }^{\mathrm{e}}$ Trima V4.

Italics illustrate values which were significantly different compared to all other systems

\section{Results}

\section{Donor Safety and Comfort}

Donor safety and comfort were analyzed in respect of PLT loss, needle time (NT), draw rate (DR), whole blood processed (WBP), and citrate infusion rate (CIR). Results obtained are summarized and statistically compared in table 3. PLT loss was lowest with TA and highest with AM, however not significantly different from the other systems ( $p \geq 0.038)$. MCS + had the significantly longest NT of all systems $(\mathrm{p}<$ 0.001). TA was the fastest machine, whereby the differences to AM and TV4 did not reach statistical significance ( $\mathrm{p} \geq 0.010)$. MCS+ demonstrated the significantly lowest DRs $(\mathrm{p}<0.001)$ as indicated by the mean blood flow (= volume of whole blood including citrate per time). DRs were similar between all other systems $(p \geq 0.010)$. SPC processed the significantly highest volumes of whole blood ( $\leq 0.001$ in DDC), while the WBP was similar with all the other systems $(p \geq 0.010)$. MCS + and SPC exposed donors to the highest amounts of anticoagulant citrate dextrose solution A (ACD-A) $(\mathrm{p} \leq 0.005)$, while equivalent values were observed among the other 3 systems $(\mathrm{p} \geq 0.010)$. When calculated as speed of ACD-A flow back to the donor, this picture changed with MCS+ (and TV4) being the lowest and TA (and SPC) the highest, proving statistical significance between these instruments $(p \leq 0.002)$. As a result, calcium was required less often with MCS+ and TV4 procedures. All citrate reactions encountered were mild and associated with return cycles only. A reduction of CIR was not required in either procedure of any system.

\section{Productivity}

Productivity was analyzed in respect of yield, volume, entire processing time $(\mathrm{PT},=$ time from set-up to the final products including priming, programming, PLT filtration of MCS+ units, PLT shaking/resuspension/transfer of AM units, product removal, splitting, sterile connection of a further storage bag for triple units), time for a standard dose (TSD 2.5), collection rate $(\mathrm{CR})$, and collection efficiency (CE). Results obtained are summarized and statistically compared in table 4. The mean volumes related to the overall collection and the 
Table 4. Productivity

\begin{tabular}{|c|c|c|c|c|c|c|}
\hline & Amicus & $\mathrm{MCS}+$ & Trima Accel & $\begin{array}{l}\text { Spectra LRS- } \\
\text { Turbo V7 }\end{array}$ & Trima V4 & $\mathrm{p}$ value \\
\hline \multicolumn{7}{|l|}{ Collection procedures, $\mathrm{n}$} \\
\hline DDC & 4 & 4 & 4 & 4 & 4 & \\
\hline TDC & 4 & 4 & 4 & 0 & 0 & \\
\hline \multicolumn{7}{|l|}{ Volume, ml } \\
\hline DDC & $484.9 \pm 4.2^{b, c, d, e}$ & $425.8 \pm 28.6^{\mathrm{a}, \mathrm{b}}$ & $467.0 \pm 7.7^{a, c, d, e}$ & $412.8 \pm 26.3^{\mathrm{a}, \mathrm{b}}$ & $424.7 \pm 8.9^{\mathrm{a}, \mathrm{b}}$ & $<0.001$ \\
\hline TDC & $684.5 \pm 14.9^{b, c}$ & $638.5 \pm 25.3^{a, b}$ & $699.8 \pm 7.7^{a, c}$ & - & - & $<0.001$ \\
\hline Unit & $237.1 \pm 9.7^{\mathrm{d}, \mathrm{e}}$ & $216.4 \pm 13.9^{\mathrm{b}}$ & $242.6 \pm 15.2^{\mathrm{c}, \mathrm{d}, \mathrm{e}}$ & $206.4 \pm 13.1^{\mathrm{a}, \mathrm{b}}$ & $212.3 \pm 4.5^{\mathrm{a}, \mathrm{b}}$ & $<0.001$ \\
\hline \multicolumn{7}{|l|}{ PLT yield $\times 10^{11}$} \\
\hline DDC & $5.68 \pm 0.22$ & $4.92 \pm 0.87$ & $5.97 \pm 0.16$ & $5.56 \pm 0.41$ & $5.67 \pm 0.64$ & 0,048 \\
\hline TDC & $8.29 \pm 0.70$ & $7.84 \pm 0.39$ & $7.66 \pm 0.70$ & - & - & 0,139 \\
\hline Unit & $2.81 \pm 0.21$ & $2.49 \pm 0.29$ & $2.76 \pm 0.31$ & $2.78 \pm 0.21$ & $2.84 \pm 0.32$ & 0,092 \\
\hline \multicolumn{7}{|l|}{ Processing time, min } \\
\hline DDC & $88.0 \pm 12.2^{\mathrm{c}}$ & $120.1 \pm 3.9^{a, b, d, e}$ & $71.1 \pm .7 .2^{\mathrm{c}, \mathrm{d}, \mathrm{e}}$ & $92.8 \pm 7.6^{\mathrm{b}, \mathrm{c}}$ & $82.4 \pm 9.6^{b, c}$ & $<0.001$ \\
\hline $\mathrm{TDC}$ & $120.0 \pm 8.5^{b, c}$ & $143.7 \pm 15.1^{a, b}$ & $92.1 \pm 15.3^{a, c}$ & - & - & $<0.001$ \\
\hline Total procedures & $104.0 \pm 10.7$ & $131.9 \pm 13.7^{\mathrm{b}, \mathrm{d}, \mathrm{e}}$ & $91.6 \pm 15.7^{c}$ & $92.8 \pm 7.6^{\mathrm{c}}$ & $82.4 \pm 9.6^{\mathrm{c}}$ & 0.001 \\
\hline Time for standard dose TSD 2.5, min & $37.7 \pm 5.5^{\mathrm{c}}$ & $54.2 \pm 11.8^{\mathrm{a}, \mathrm{b}, \mathrm{e}}$ & $30.2 \pm 5.6^{\mathrm{c}, \mathrm{d}}$ & $42.0 \pm 5.9^{\mathrm{b}}$ & $36.9 \pm 7.2^{\mathrm{c}}$ & $<0.001$ \\
\hline \multicolumn{7}{|l|}{ Collection rate, $\times 10^{9} / \mathrm{min}$} \\
\hline DDC & $9.8 \pm 1.9^{\mathrm{c}, \mathrm{d}}$ & $4.9 \pm 0.9^{a, b, d, e}$ & $10.7 \pm 1.2^{\mathrm{c}, \mathrm{d}, \mathrm{e}}$ & $7.3 \pm 1.0^{\mathrm{a}, \mathrm{b}, \mathrm{c}}$ & $8.4 \pm 1.5^{\mathrm{b}, \mathrm{c}}$ & $<0.001$ \\
\hline TDC & $10.4 \pm 1.2^{\mathrm{c}}$ & $7.1 \pm 1.0^{a, b}$ & $11.2 \pm 1.3^{\mathrm{c}}$ & - & - & 0.003 \\
\hline Total procedures & $10.0 \pm 1.5^{\mathrm{c}, \mathrm{d}}$ & $6.0 \pm 1.5^{\mathrm{a}, \mathrm{b}}$ & $10.9 \pm 2.1^{\mathrm{c}, \mathrm{d}}$ & $7.3 \pm 1.0^{\mathrm{a}, \mathrm{b}}$ & $8.4 \pm 1.5$ & $<0.001$ \\
\hline \multicolumn{7}{|l|}{ Collection efficiency, \% } \\
\hline DDC & $71.3 \pm 0.4^{\mathrm{b}, \mathrm{d}}$ & $59.6 \pm 8.8^{b}$ & $76.6 \pm 0.9^{a, c, d, e}$ & $53.9 \pm 4.8^{\mathrm{a}, \mathrm{b}, \mathrm{e}}$ & $67.6 \pm 6.7^{\mathrm{b}, \mathrm{d}}$ & $<0.001$ \\
\hline TDC & $70.9 \pm 5.9$ & $68.4 \pm 3.1$ & $72.4 \pm 4.8$ & - & - & 0.053 \\
\hline Total procedures & $71.1 \pm 3.9^{\mathrm{c}, \mathrm{d}}$ & $64.0 \pm 3.9^{\mathrm{b}}$ & $74.5 \pm 3.9^{\mathrm{c}, \mathrm{d}}$ & $53.9 \pm 4.8^{\mathrm{a}, \mathrm{b}}$ & $67.6 \pm 6.7$ & $<0.001$ \\
\hline
\end{tabular}

DDC $=$ Double-dose PLT collection $;$ TDC $=$ triple-dose PLT collection; $\mathrm{PLT}=$ platelet.

Significant to: ${ }^{\mathrm{a}}$ Amicus, ${ }^{\mathrm{b}}$ Trima Accel, ${ }^{\mathrm{C}} \mathrm{MCS}+,{ }^{\mathrm{d}}$ Spectra LRS-Turbo V7, ${ }^{\mathrm{e}}$ Trima V4.

Italics illustrate values which were significantly different compared to all other systems.

Volume and PLT yield of MCS+ units were obtained after filtration.

single units were highest with $\mathrm{AM}$ and TA demonstrating significant differences to the 3 other systems $(\mathrm{p} \leq 0.002)$ with similar values $(p \geq 0.007)$. No significant differences were observed with PLT yield ( $p \geq 0.021$ ). All units surpassed the minimum European single unit PLT yield of $2.0 \times 10^{11}$ [14]. As observed with NT, PT was significantly longest with MCS+ and significantly shortest with TA ( $\mathrm{p} \leq 0.005$ in DDC). TA was the fastest in obtaining an established standard dose and demonstrated significant differences to MCS+ and SPC ( $\mathrm{p} \leq 0.005$ ) with the highest values, while AM and TV4 were similar $(\mathrm{p}=0.574)$. TA and AM demonstrated the highest CRs with significant differences to MCS + and SPC ( $p \leq 0.005)$ presenting the lowest values, while TV4 took an interim position. As expected from the differences in $\mathrm{CR}, \mathrm{CE}$ was highest with TA (significantly higher to all other systems during DDC, $\mathrm{p}<0.001$ ), while AM was a close second. SPC was less efficient than the other systems, while MCS+ and TV4 were simi$\operatorname{lar}(\mathrm{p}=0.505)$.

\section{In vitro Quality}

In vitro quality was analyzed for rest cell contamination, lactate and glucose content, ristocetin-induced aggregation, HSR, and CD62P expression. Results obtained are summa- rized and statistically compared in table 5 . All products were sufficiently leukoreduced to below $1 \times 10^{6}$ WBCs. Contamination with WBCs was highest with AM and lowest with Trima PLTs, proving statistical significance between these systems ( $\mathrm{p} \leq 0.005$ ). RBC contamination was similar among the study groups $(p \geq 0.010)$ and met the European threshold of $\leq 3.0 \times 10^{9}[14]$. The highest initial $\mathrm{pH}$ values were observed with the GAMBRO systems, proving statistical significance to AM with the significantly lowest initial values of all systems $(\mathrm{p}<0.001)$. Differences in $\mathrm{pH}$ values became equivalent during 5 days of storage. Throughout the entire storage period, AM PLTs demonstrated the highest CD62P expression with significant differences to Trima PLTs ( $\mathrm{p} \leq 0.001)$, while CD62P expression of MCS+ and SPC PLTs was equivalent ( $\mathrm{p}=$ 0.678). All PLT units demonstrated similar values for glucose consumption and lactate production and were equivalent for hypotonic shock response and ristocetin-induced aggregation.

\section{Discussion}

Multiple-dose PLT apheresis can contribute to providing sufficient blood supply in a situation of limited human resources. 
Table 5. Rest cell contamination and storage-related product characteristics

\begin{tabular}{|c|c|c|c|c|c|c|}
\hline & Amicus & $\mathrm{MCS}+$ & Trima Accel & $\begin{array}{l}\text { Spectra LRS- } \\
\text { Turbo V7 }\end{array}$ & Trima V4 & $\mathrm{p}$ value \\
\hline Units examined, $\mathrm{n}$ & 20 & 20 & 20 & 16 & 16 & \\
\hline \multicolumn{7}{|l|}{ WBCs $\log 10 /$ unit } \\
\hline All units & $5.36 \pm 0.10^{\mathrm{b}, \mathrm{e}}$ & $5.17 \pm 0.23$ & $4.92 \pm 0.42^{\mathrm{a}}$ & $5.21 \pm 0.34$ & $4.87 \pm 0.25^{\mathrm{a}}$ & 0.002 \\
\hline \multicolumn{7}{|l|}{ RBCs $\times 10^{9} /$ unit } \\
\hline $\begin{array}{l}\text { All units } \\
\text { pH value }\left(22^{\circ} \mathrm{C}\right)\end{array}$ & $0.75 \pm 0.34$ & $0.45 \pm 0.28$ & $0.66 \pm 0.70$ & $0.45 \pm 0.22$ & $0.45 \pm 0.13$ & 0.175 \\
\hline Day 1 & $7.31 \pm 0.04^{b, c, d, e}$ & $7.44 \pm 0.06^{\mathrm{a}}$ & $7.52 \pm 0.03^{\mathrm{a}}$ & $7.48 \pm 0.04^{\mathrm{a}}$ & $7.51 \pm 0.04^{\mathrm{a}}$ & $<0.001$ \\
\hline Day 5 & $7.32 \pm 0.08$ & $7.34 \pm 0.12$ & $7.48 \pm 0.11$ & $7.38 \pm 0.07$ & $7.43 \pm 0.11$ & 0.018 \\
\hline \multicolumn{7}{|l|}{ CD62P, \% } \\
\hline Day 1 & $38.8 \pm 7.6^{\mathrm{b}, \mathrm{e}}$ & $28.9 \pm 7.9$ & $18.6 \pm 5.1^{\mathrm{a}, \mathrm{d}}$ & $29.4 \pm 9.9^{\mathrm{b}}$ & $22.8 \pm 9.7^{\mathrm{a}}$ & 0.001 \\
\hline Day 5 & $58.9 \pm 4.5^{\mathrm{b}, \mathrm{e}}$ & $56.2 \pm 4.5^{\mathrm{b}}$ & $42.6 \pm 4.0^{\mathrm{a}, \mathrm{c}}$ & $53.7 \pm 14.4$ & $49.0 \pm 5.3^{\mathrm{a}}$ & 0.018 \\
\hline \multicolumn{7}{|l|}{ Aggregation, \% } \\
\hline Day 1 & $92.1 \pm 4.6$ & $95.2 \pm 2.3$ & $92.6 \pm 4.0$ & $94.1 \pm 3.8$ & $94.9 \pm 3.8$ & 0.416 \\
\hline Day 5 & $88.8 \pm 4.3$ & $93.4 \pm 3.9$ & $90.6 \pm 2.4$ & $89.3 \pm 3.4$ & $90.2 \pm 3.5$ & 0.136 \\
\hline \multicolumn{7}{|l|}{ HSR, \% } \\
\hline Day 1 & $84.0 \pm 3.9$ & $83.5 \pm 5.7$ & $91.0 \pm 5.5$ & $84.7 \pm 7.2$ & $84.3 \pm 3.3$ & 0.024 \\
\hline Day 5 & $84.3 \pm 6.2$ & $81.0 \pm 4.1$ & $81.8 \pm 4.9$ & $77.2 \pm 6.4$ & $78.5 \pm 6.2$ & 0.125 \\
\hline \multicolumn{7}{|c|}{ Glucose consumption, $\times 10^{-12} \mathrm{mg} /$ PLT } \\
\hline Days 1-3 & $3.31 \pm 0.71$ & $3.28 \pm 1.74$ & $2.64 \pm 0.41$ & $3.10 \pm 0.96$ & $2.74 \pm 0.47$ & 0.361 \\
\hline Days 3-5 & $2.52 \pm 1.03$ & $1.80 \pm 0.86$ & $2.70 \pm 0.53$ & $2.70 \pm 0.80$ & $2.83 \pm 0.82$ & 0.174 \\
\hline \multicolumn{7}{|c|}{ Lactate production, $\times 10^{-12} \mathrm{mmol} / \mathrm{PLT}$} \\
\hline Days 1-3 & $3.47 \pm 0.65$ & $3.52 \pm 1.06$ & $2.57 \pm 0.55$ & $3.24 \pm 0.68$ & $3.11 \pm 0.57$ & 0.094 \\
\hline Days $3-5$ & $2.52 \pm 1.13$ & $2.57 \pm 0.51$ & $2.77 \pm 0.56$ & $2.67 \pm 0.98$ & $2.99 \pm 0.74$ & 0.615 \\
\hline
\end{tabular}

WBCs = white blood cell; RBCs = red blood cells; PLT = platelet; HSR $=$ hypotonic shock response

Significant to: ${ }^{\mathrm{a}} \mathrm{Amicus},{ }^{\mathrm{b}}$ Trima Accel, ${ }^{\mathrm{c}} \mathrm{MCS}+,{ }^{\mathrm{d}}$ Spectra LRS-Turbo V7, ${ }^{\mathrm{e}}$ Trima V4.

Glucose consumption $\left(\times 10^{-12} \mathrm{mg}\right)$ and lactate production $\left(\times 10^{-12} \mathrm{mmol}\right)$ per PLT were calculated from the measured supernatant contents of glucose $(\mathrm{mg} / \mathrm{dl})$ and lactate $(\mathrm{mmol} / \mathrm{l})$ divided by the mean PLT count $\left(\times 10^{9} / \mathrm{l}\right)$.

The collection of several PLT units from the same donor may especially be of benefit in bone marrow aplasia following chemotherapy or stem cell transplantation. To investigate whether new devices can combine higher efficiencies with shorter processing times, a prospective crossover comparison between newly introduced systems and current equipment was conducted. A short overall processing time might increase the collection of products per time unit and improve donor comfort by lowering the volume of processed whole blood and citrate load. A valid comparison should be independent of donor or center immanent factors. This was attained by performing all collections using the same staff and donors as well as similar definitions and target endpoints on each instrument. Confirming previous reports [16, 21, 25], PLT loss was lowest with TA and highest with AM. The latter, however, returned additional saline solution that could have caused higher dilution of the donors' peripheral blood, thus mimicking an increased PLT loss. Apart from PLT loss, DR and the volume of WBP are other variables important for donor comfort. The drawback with higher DRs and increased WBP volumes is greater demand on access and greater potential for citrate reactions. While all machines processed similar but considerably lower amounts of whole blood than SPC, lower DRs were ob- served with MCS+ only. As reflected by the lower frequency of administered calcium, donors sensitive to citrate may profit from these lower DRs, coupled with the lowest CIRs of all systems. As shown recently in a national audit of citrate toxicity in PLT apheresis donors [7], the BAXTER and GAMBRO systems showed significantly more citrate incidents as compared to MCS+. Additionally, Bueno et al. [25] reported that donors who attached importance to comfort, preferred the $\mathrm{MCS}+$ machine. From the experience of Zingsem et al. [10, $16,26,27]$ citrate reactions very rarely occur at CIRs of up to $1.1 \mathrm{ml} \mathrm{ACD}-\mathrm{A} / \mathrm{min} / \mathrm{l}$. Despite our small study size, we would rather recommend CIRs reduced to a 'MCS+ like level' of $0.80-0.90 \mathrm{ml} / \mathrm{min} / \mathrm{l}$ to further improve donor comfort.

By splitting yields from single-donor PLT apheresis sessions into 2 or more components, blood suppliers respond to decreasing financial resources and donor pools and concurrently increasing demands for PLTs. For splitting blood products, higher collection volumes must be attained, which should also be taken into account when analyzing donor comfort/safety. According to German guidelines, the plasma volume donated per year should not exceed 28.51 [13]. This limit is far from being reached, even in triple-dose PLT donors, when keeping to a collection volume of about $700-750 \mathrm{ml}$ (as performed in 
our study) and a donation-free interval of 4-6 weeks (as performed in our blood bank routine). On the other hand, splitting of blood products may result in PLT dose reduction (in our study: $0.3 \times 10^{11}$ less PLTs in TDC as compared to DDC) that could be of clinical impact, as several investigations reported significantly higher post-transfusion count increments and longer transfusion-free intervals with increasing PLT doses [28, 29]. Further enhancement of collection volumes may overcome this problem but would influence donor safety/comfort. Thus, donation criteria and the optimal length of the donation-free interval should be evaluated in larger study sizes, which probably may lead to changes in guidelines and regulatory approval for TDC.

The new devices investigated were equally or even more efficient than our current equipment, especially SPC. The CEs noted complied favorably with the data reported in the literature for TA $(71-76 \%)$ [16, 30], AM (66-73\%) [18, 31-33], MCS + (60-63\%) [33, 34], TV4 (63-65\%) [16, 35], and SPC $(51-56 \%)[18,35,36]$. Because CE depends markedly on preprocedure donor characteristics irrespective of procedure time, CR probably appears to be a more practical way of comparing PLT apheresis systems because it addresses both, PLT yield and time, considered today as important as yield when evaluating equipment. In a recent report, a short NT was the most highly valued measure for donors when choosing a cell separator, and, as a result, crucially important for donor recruitment and retention [25]. Therefore, the higher CRs of $\mathrm{AM}$ and TA in particular leading to shorter NTs compared to our current equipment must be considered as real improvement, whereas the lower CRs and longer NTs of MCS+ procedures were a real drawback as also described previously [37]. This was most likely explained by the discontinuous separation technique, which is a profound difference in performance compared to all the other systems which operate continuously. While NT directly concerns apheresis donors, PT is important for operators to calculate the maximal possible number of units per day. As observed with NT, PT was longest with MCS+ and shortest with TA, while AM, though faster in NT, was equivalent to our current equipment. This was mainly the result of the fact that AM PLTs necessitated manual resuspension and shaking in PLT-poor plasma generated from the machine prior to transfer into the final storage bags. This caught the operator's attention, and the machine was blocked for further collections. As calculated from TSD 2.5 (table 4), only TA allowed for additional units per machine per 8-hour working day compared to our current equipment. Another drawback of AM procedures consisted in higher PLT activation, also reported by Hagberg et al. [38]. While PLTs harvested with MCS+, TA, TV4, or SPC were transferred intermittently or continuously to large PLT storage containers outside the centrifuge, AM PLTs were stored as 'dry PLTs' in a small bag within the centrifuge during the entire separation procedure, resulting in intimate contact with each other and the plastic material. Together with vigorous handling required for resuspension, this could have caused higher degrees of PLT activation and probably lower initial $\mathrm{pH}$ values. As $\mathrm{p}$-selectin has been shown to be the PLT receptor for WBC binding [39], the higher CD62P expression of AM PLTs could also explain the higher WBC contamination which is significant compared to Trima PLTs with the lowest initial CD62P expression. This finding was also described previously upon comparison of AM vs. GAMBRO PLTs [11]. Adhesion between activated PLTs and WBCs results in WBC activation [40] which probably in turn accelerates secretion of pro-inflammatory cytokines and consequently strengthens adverse transfusion reactions [38].

In summary, TA was faster in obtaining an established standard dose compared to our current equipment, and, because of this advantage, is the machine with the highest potential to optimize productivity in routine use. Due to its discontinuous separation technique, MCS+ demonstrated lower collection rates leading to significantly longer procedure durations which were, however, compensated with fewer citrate side reactions resulting from significantly lower CIRs and DRs. AM lost its advantage of a quick donation to an increased manual workload from donor disconnection to the final products, resulting in long overall procedure times and increased PLT activation. This might be of clinical relevance and should probably be reconsidered for further improvement.

\section{References}

1 Zingsem J, Moog R: Preparative haemapheresis and donor safety. Transfus Med Hemother 2004;31: $5-6$.

2 Moog R: Implementation of concurrent red blood cell and platelet collection by apheresis in a university haemapheresis unit. Transfus Med 2004;14: 145-150.

3 Snyder EL, Elfath MD, Taylor H, Rugg N, Greenwalt TJ, Baril L, Whitley P, Brantigan B, Story K: Collection of two units of leukoreduced RBCs from a single donation with a portable multiplecomponent collection system. Transfusion 2003;43: 1695-1705.

4 Valbonesi M, Giannini G, Morelli R, Frisoni R, Capra C: Multicomponent collection as of 2005. Transfus Apher Sci 2005;32:287-297.
5 Popovsky MA: Multicomponent apheresis blood collection in the United States: current status and future directions. Transfus Apher Sci 2005;32: 299-304.

6 Popovsky MA: Complications of blood donation: manual and automated collection procedures. Transfus Med Hemother 2004;31:49-53.

7 Makar YF, Butler MO, Cockersole GM, Gabra G, Serevitch JM: National audit of citrate toxicity in plateletpheresis donors. Transfus Med 2002;12: 187-191.

8 Fournel JJ, Zingsem J, Riggert J, Muylle L, Müller $\mathrm{N}$, Kohler M, Beaumont JL, Baeten M, Eckstein R, van Waeg G: A multicenter evaluation of the routine use of a new white cell-reduction apheresis system for collection of platelets. Transfusion 1997;37: 487-492.
9 Zingsem J, Glaser A, Weisbach V, Zimmermann R, Neidhardt B, van Waeg G, Eckstein R: Evaluation of a platelet apheresis technique for the preparation of leukocyte-reduced platelet concentrates. Vox Sang 1998;74:189-192.

10 Zingsem J, Zimmermann R, Weisbach V, Glaser A, Bunkens H, Eckstein R: Comparison of a new WBC-reduction system and the standard plateletpheresis protocol in the same donors. Transfusion 2001;4:396-400.

11 Stiegler G, Leitner G, Jurko S, Gerhartl K, Höcker $\mathrm{P}$ : Quality control in apheresis platelet concentrates: various WBC reduction technologies lead to different leukocyte depletion. Transfus Med Hemother 2003;30:299-300 P7 (abstract). 
12 Schlenke P: Leukocyte reduction in blood component supply: the impact of flow cytometry in assessing residual leukocytes. Transfus Med Hemother 2005;32:12-19.

13 German Medical Association and Paul-Ehrlich-Institute: Guidelines for the Collection of Blood and Blood Components and the Usage of Blood Products (Hemotherapy), rev ed. Cologne, Deutscher Ärzteverlag, 2005.

14 Council of Europe: Guide to the Preparation, Use and Quality Assurance of Blood Components, 11th ed. Strasbourg, Council of Europe Press, 2005.

15 Performance of preparative cellular hemapheresis for the collection of blood cell concentrates. II Recommendations of the German Society for Transfusion Medicine and Immunohematology for preparative white cell and plateletpheresis. Infus Ther Transfus Med 1998;25:376-382.

16 Ringwald J, Zingsem J, Zimmermann R, Strasser E, Antoon M, Eckstein R: First comparison of productivity and citrate donor load between the Trima version 4 (dual-stage-filler) and the Trima Accel (single-stage-filler) in the same donors. Vox Sang 2003;85:267-275.

17 Burgstaler EA, Pineda AA, Potter BM, Brown R: Plateletapheresis with a next generation blood cell separator. J Clin Apher 1997;12:55-62.

18 Burgstaler EA, Pineda AA, Bryant SC: Prospective comparison of plateletapheresis using four apheresis systems on the same donors. J Clin Apher 1999;14:163-170.

19 Valbonesi M, Florio G, Ruzzenenti MR, Bo A, Bruni R, Giannini G, Morelli F: Multicomponent collection (MCC) with the latest hemapheresis apparatuses. Int J Artif Organs 1999;22:511-515.

20 Menichella G, Serafini R, Ciarli M, Paladini U, Pierelli L, Bunkens H, Leone G: A new blood donation strategy: automated blood collection (ABC). Int J Artif Organs 2001;24:173-177.
21 Moog R, Bartsch R, Müller N: Concurrent collection of in-line filtered platelets and red blood cells by apheresis. Ann Hematol 2002;81:322-325.

22 Picker SM, Speer R, Gathof BS: Functional characteristics of buffy-coat platelets photochemicallytreated with amotosalen- $\mathrm{HCl}$ for pathogen inactivation. Transfusion 2004;44:320-329.

23 Fantl P: Osmotic stability of blood platelets. J Physiol 1968;198:1-16.

24 Born GVR, Cross MJ: The aggregation of blood platelets. J Physiol 1963;168:178-195

25 Bueno JL, Garcia F, Castro E, Barea L, Gonzalez $\mathrm{R}$ : A randomized crossover trial comparing three plateletpheresis machines. Transfusion 2005;45: 1373-1381.

26 Zingsem J, Zimmermann R, Weisbach V, Glaser A, van Waeg $G$, Eckstein R: Comparison of COBE white cell-reduction and standard plateletpheresis protocols in the same donors. Transfusion 1997;37: 1045-1049.

27 Zingsem J, Weisbach V, Zimmermann R, Glaser A, Bunkens H, Eckstein R: Preparation of fresh frozen plasma as a by-product of plateletpheresis. Transfusion 2002;42:81-86.

28 Norol F, Bierling P, Roudot-Thoraval F, Ferrer Le Cœur F, Rieuw C, Lavaux A, Kuentz M, Duedari $\mathrm{N}$ : Platelet transfusion. A dose-response study. Blood 1998;92:1448-1453.

29 Klumpp TR, Herman JH, Gaghan JP, Russo RR, Christman RA, Goldberg SL, Ackerman SJ, Bleecker GC, Mangan KF: Clinical consequences of alterations in platelet transfusion dose: a prospective, randomized, double-blind trial. Transfusion 1999;39:674-681.

30 Burgstaler EA, Winters JL, Pineda AA: Paired comparison of Gambro Trima Accel versus Baxter Amicus single-needle plateletpheresis. Transfusion 2004;44:1612-1620.
31 Benjamin RJ, Rojas P, Christmas S, Neal J, Broughton S, Burgio C, Barrett B, Churchill WH: Plateletapheresis efficiency: a comparison of the Spectra LRS and AMICUS separators. Transfusion 1999;39:895-899.

32 Moog R, Müller N, Goergens D: Platelet collection with the Amicus and AS.TC 204 blood cell separators. Transfusion 1998;38:285-289.

33 Moog R, Müller N: White cell reduction during plateletpheresis: a comparison of three blood cell separators. Transfusion 1999;39:572-577.

34 Rehacek V, Samkova H: Comparison of the LDP C5 and LDP C2 protocols for platelet concentrates preparation on the Haemonetics MCS+ separator. Transfus Med Hemother 2003;30:298 P3 (abstract).

35 Bueno JL, Barea L, Garcia F, Castro E: A comparison of PLT collections from two apheresis devices. Transfusion 2004;44:119-124.

36 Strasser EF, Schuster M, Egler K, Bauer J, Weisbach V, Ringwald J, Zimmermann R, Zingsem J, Eckstein R: Frequently used plateletpheresis techniques result in variable target yields and platelet recruitment of donors. Transfusion 2005;45: 788-797.

37 González-Bachs A, Pinacho AA: Results of a productivity comparison of Trima ${ }^{\circledR}$ Accel $^{\mathrm{TM}}$ with Haemonetics-MCS+ in platelet collections. Transfus Med Hemother 2003;30:298-299(abstract).

38 Hagberg IA, Akkøk CA, Lyberg T, KjeldsenKragh J: Apheresis-induced platelet activation: comparison of three types of cell separators. Transfusion 2000;40:182-192.

39 Rinder HM, Bonan JL, Rinder CS, Ault KA, Smith BR: Dynamics of leukocyte-platelet adhesion in whole blood. Blood 1991;78:1730-1737.

40 Neumann FJ, Marx N, Gawaz M, Brand K, Ott I, Rokitta C, Sticherling C, Meinl C, May A, Schomig A: Induction of cytokine expression in leukocytes by binding to thrombin-stimulated platelets. Circulation 1997;95:2387-2394. 